\title{
Shrinkage Estimator for Bayesian Network Parameters
}

\author{
John Burge and Terran Lane \\ University of New Mexico, New Mexico, USA \\ \{lawnguy, terran\} @ CS. unm. edu
}

\begin{abstract}
Maximum likelihood estimates (MLEs) are commonly used to parameterize Bayesian networks. Unfortunately, these estimates frequently have unacceptably high variance and often overfit the training data. Laplacian correction can be used to smooth the MLEs towards a uniform distribution. However, the uniform distribution may represent an unrealistic relationships in the domain being modeled and can add an unreasonable bias. We present a shrinkage estimator for domains with hierarchically related random variables that smoothes MLEs towards other distributions found in the training data. Our methods are quick enough to be performed during Bayesian network structure searches. On both a simulated and a real-world neuroimaging domain, we empirically demonstrate that our estimator yields superior parameters in the presence of noise and greater likelihoods on left-out data.
\end{abstract}

Keywords: Bayesian networks, shrinkage, hierarchy, parameter estimation.

\section{Introduction}

Our primary contribution is a shrinkage parameter estimator that allows learned Bayesian networks (BNs) to be less affected by noisy data and to generalize more effectively to unseen data than maximum likelihood estimates (MLEs) with Laplace smoothing. We also demonstrate that the estimator operates quickly enough to be performed during Bayesian network structure searches.

The amount of data available to train a BN's parameters is often inadequate to train MLEs, resulting in high variance estimates that overfit the data and generalize poorly to left-out data. These effects can be lessened by smoothing the MLE towards distributions with lower variance. One widely employed method is Laplace smoothing [7] which smoothes MLEs towards a uniform distribution. While this has been shown to improve BN classification [7], the uniform distribution is an arbitrary choice that may represent an unrealistic bias in complex real-world domains.

We propose smoothing MLEs towards other distributions found in the data. To illustrate our method, we will start with an example in a neuroscience domain. Assume that the correlation between the visual cortex and the motor cortex is being modeled for some population of mentally-ill patients. For discrete BNs, this relationship can be parameterized with a series of multinomials. The MLEs for these multinomials will have a certain degree of variance and may overfit the data. Laplacian smoothing helps, but introduces a bias that is not observed in the data. Uniform distributions poorly model relationships between brain regions. 
Instead, we can smooth towards other distributions in the data. In particular, we can take advantage of the anatomical placement of the cortices within larger regions of the brain. The visual cortex is encased in the occipital lobe and the motor cortex is encased in the frontal lobe. Since the lobes contain the cortices, the relationship between the lobes may be similar to the relationship between the cortices. Thus, we propose smoothing MLEs for the cortices towards MLEs for the lobes. There is more training data available for the lobes than for the cortices (they are larger structures) and thus, their MLEs will be lower in variance. This smoothing still introduces some bias, but it is more realistic than the bias added by Laplacian smoothing.

Smoothing MLEs towards other "similar" MLEs with lower variance is often referred to as shrinkage and has been successfully employed in Machine Learning applications (Section 1.2). What makes two MLEs "similar" depends on the domain. In the neuroscience domain, two MLEs are said to be "similar" if they are associated with correlations between brain regions that contain or are contained by each other. In previous applications [14], MLEs were "similar" if they were associated with the number of times a particular word appeared in different classes of text documents. We formalize the notion of "similarity" as a hierarchical arrangement of the random variables (RVs) in a domain. This hierarchy is then used to select distributions that are shrunk towards when computing MLEs.

We compare the performance of BNs trained with our shrinkage estimators versus BNs trained with MLEs plus Laplace smoothing (MLE+L) on two sets of data: a simulated data domain, where ground truth is known and controllable and a set of ten real-world neuroimaging datasets. On both the simulated and neuroimaging datasets, we find that shrinkage estimates are more robust to noisy data. On the neuroimaging datasets, we show that shrinkage estimates increase the likelihood of BNs on data left out of the training process. While classification is not the main focus of this work, we also demonstrate that shrinkage estimators do not degrade the classification performance of BN-based classifiers.

\section{Background}

\subsection{Bayesian Networks}

BNs [15] are graphical models that explicitly represent dependencies among RVs. A BN's topological structure, represented as a directed acyclic graph (DAG), contains nodes for RVs and directed links between correlated parent and child nodes. A family is composed of a single child and its parents. We assume fully observable discrete RVs so that a family's conditional probability, $P$ (child I parents), can be represented with a conditional probability table (CPT).

Searching for a BN's topology is accomplished by proposing many hypothesis structures guided by a search heuristic (often an iterative hill-climbing heuristic), while measuring how well each structure corresponds to correlations in the data via a structure scoring function. Common scores include MDL [13], BDe [10], conditional likelihood (CL) [8], etc. Some scores require a BN's parameters to be trained (e.g., MDL and CL), but others marginalize out the parameters (e.g., BDe). 
We will use the following notation. Let $\boldsymbol{X}$ be a set of $n \mathrm{RVs},\left\{X_{1}, X_{2}, \ldots, X_{n}\right\}$, with arities $r_{l}, r_{2}, \ldots, r_{n}$. A data point is a fully observable assignment of values to $X$. A $\mathrm{BN}, B$, over $\boldsymbol{X}$ is described by the pair $\left\langle B_{S}, B_{\Theta}\right\rangle . B_{S}$ is the DAG representing the BN's structural topology. $B_{\theta}=\left\{\theta_{X_{i}, j, k}: 1 \leq i \leq n, 1 \leq j \leq q_{i}, 1 \leq k \leq r_{i}\right\}$ is the set of parameters where $\theta_{X_{i}, j, k}=P\left(X_{i}=k \mid P a\left(X_{i}\right)=j\right)$. $\quad X_{i}$ 's parent set is denoted $\operatorname{Pa}\left(X_{i}\right) . q_{i}$ is the number of configurations for the RVs in $P a\left(X_{i}\right)$. Depending on context, " $X_{i}$ " will also refer to the family with child $\mathrm{RV} X_{i}$.

\subsection{Shrinkage}

For any system of at least three parameters, Stein [16] demonstrated there exists an estimator with lower quadratic risk than the MLE, where risk is measured as the expected difference between the true parameters and the estimated parameters. This holds even when the parameters are independently distributed. James and Stein [11] introduced such an estimator, the acclaimed James Stein Estimator (JSE), for normally distributed RVs with identity covariance matrices. The JSE shrinks the MLE towards a value computed from all the parameters being estimated. The inclusion of this bias reduces the estimator's risk. Similar results have been extended to the multinomial distributions that are used in discrete BNs [9]. The JSE also played a central role in empirical Bayesian methods in general [5].

McCallum et al. [14] demonstrated that shrinkage could also be applied to text document classification given hierarchically related document classes. Instead of shrinking the MLE estimate toward a single value (as done by Stein), the parameter estimate is shrunk towards a combination of estimates across multiple classes of data,

$$
\breve{\theta}=\lambda^{0} \hat{\theta}^{0}+\lambda^{1} \hat{\theta}^{1}+\ldots+\lambda^{n} \hat{\theta}^{n}
$$

where $\breve{\theta}$ is the shrinkage estimate, $\hat{\theta}^{i}$ is the estimate for the $i^{\text {th }}$ class's MLE and $\lambda^{i}$ is a linear mixing weight for the $i^{\text {th }}$ class. The weights were set via an expectation maximization (EM) algorithm. McCallum et al. demonstrated increased classification performance when using shrinkage estimates relative to MLEs. Anderson, Domingos and Weld demonstrated how this form of shrinkage could also be applied in Relational Markov models [1]. They used a different method to learn mixture weights, but still found significant increases in model performance.

Building on the previous success of this form of shrinkage, we demonstrate how shrinkage can be applied among RVs within the same class of data. This allows shrinkage to be applied even when there are not hierarchically related classes of data, but instead hierarchically related RVs within the same class.

\subsection{Random Variable Aggregation Hierarchies}

There are many domains that have a natural hierarchical decomposition of RVs. For example, image analyses, where pixel neighborhoods of varying size can be aggregated together; genetic regulatory network reconstruction, where genes can be decomposed into families and super-families; word types in grammar trees; medical diagnoses, where diseases and symptoms are grouped into sub-categories; Fourier and wavelet analyses, where coefficients are spatially and temporally related; etc. 


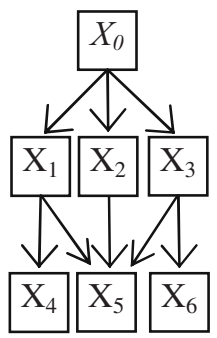

a)

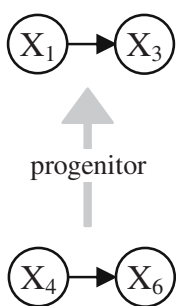

b)

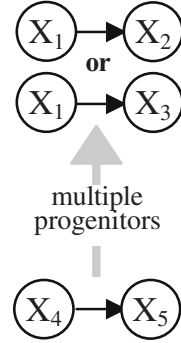

c)
Fig. 1. a) Hypothetical trellis hierarchy. (this is not a $\mathrm{BN}$ ). b) $X_{4} \rightarrow X_{6}$ family and its progenitor. c) $X_{4} \rightarrow X_{5}$ family and its multiple valid progenitors.
Start: Initialize coefficients

$$
\lambda_{X_{i}, j}^{p}=1 /\left(V_{i}+1\right)
$$

'E' step: Gauge ability for CPTs to predict events in $\widetilde{D}^{\prime}$

$$
\begin{aligned}
\beta_{X_{i}, j}^{p} & =\sum_{d \in \widetilde{D}^{\prime}} P\left(\hat{\theta}_{X_{i}, j}^{p} \text { generated } d\right) \\
& =\sum_{k=1}^{r_{i}} \frac{\lambda_{X_{i}, j}^{p} \widehat{\theta}_{X_{i}, j, k}^{p}}{\sum_{m=1}^{V_{i}} \lambda_{X_{i}, j}^{m} \hat{\theta}_{X_{i}, j, k}^{m}} N_{X_{i}, j, k}^{\widetilde{D}^{\prime}}
\end{aligned}
$$

'M' step: Calculate guaranteed improved weights by normalizing $\beta$ 's.

$$
\lambda_{X_{i}, j}^{p}=\beta_{X_{i}, j}^{p} / \sum_{m=1}^{V_{i}} \beta_{X_{i}, j}^{m}
$$

Fig. 2. Coefficient learning algorithm

In the neuroimaging domain we demonstrate our methods on, there is a hierarchical relationship among neuroanatomical brain regions. Each brain region decomposes into a set of smaller brain regions and the activation associated with each brain region is calculated as the average weighted activation of the brain regions it comprises. From these hierarchical relationships, we build a hierarchy such that each node in the hierarchy corresponds to a brain region. The parents of a node in the hierarchy (referred to as an h-parent since parent describes relationships in BNs) correspond to brain regions the node is part of and children of a node in the hierarchy (h-children) are the regions the node is composed of.

A RV hierarchy is composed of composite and atomic RVs. The atomic RVs exist at the bottom level of the hierarchy and have no h-children. They correspond to the non-decomposable elements in the domain. In the neuroimaging domain, the smallest regions of the brain are represented as atomic RVs. Composite RVs have h-children and their values can be computed from their h-children via an aggregation function.

Let $\boldsymbol{X}$ contain both the atomic and composite RVs. Let $\hat{\boldsymbol{X}}$ be a subset of $\tau$ RVs in $\boldsymbol{X},\left\{X_{1}, \ldots, X_{\tau}\right\}$. An aggregation function $Y=\xi(\hat{\boldsymbol{X}})$, is a scalar function of $\hat{\boldsymbol{X}}$ where $Y$ is a composite RV whose distribution reflects an aspect of the joint distribution of the RVs in $\hat{\boldsymbol{X}}$. We use the weighed mean aggregation function in our experiments. Thus, a composite RV's value is the weighted mean of its h-children's values (this is a slight simplification as the weighted mean must be discretized).

A hierarchy over the RVs in $\boldsymbol{X}$, denoted as $\Lambda$, can be graphically represented as a trellis (Figure 1a). A trellis is a relaxation of a forest such that each node may have multiple parents. Let $\Lambda_{X_{i}}$ denote the h-children of $X_{i}, \Lambda^{X_{i}}$ denote the h-parents of $X_{i}$ and level ${ }_{\Lambda}\left(X_{i}\right)$ denote the integer-valued level $X_{i}$ is located in. $\Lambda_{X_{i}}=\varnothing$ for leaves and $\Lambda^{X_{i}}=\varnothing$ for the $\operatorname{root}(\mathrm{s})$. If $X_{i}$ is not a leaf node then $X_{i}=\xi\left(\Lambda_{X_{i}}\right)$. An h-level corresponds to all RVs at one level in the hierarchy. 


\subsection{Calculating BN Parameters with Shrinkage}

To demonstrate how to calculate a BN's parameters with shrinkage, assume we are computing the parameters for a BN family containing a $\mathrm{RV} X_{4}$ as a parent and $\mathrm{RV} X_{6}$ as a child and we need a distribution to smooth this family's MLE towards. To find this distribution, we can look to the hierarchy the RVs are in. Assume this is the hierarchy given in Figure 1a. $X_{4}$ 's and $X_{6}$ 's h-parents are $X_{1}$ and $X_{3}$, respectively.

Since $X_{I}$ 's value is partially determined by $X_{4}$ 's value, the probability distribution for $X_{I}$ is likely to be similar to the distribution for $X_{4}$. Likewise, $X_{3}$ 's distribution is likely to be similar to $X_{6}$ 's. Further, the CPT detailing the correlation between $X_{1}$ and $X_{3}$ may also be similar to the CPT detailing the correlation between $X_{4}$ and $X_{6}$. Thus, we propose using the CPT in the $X_{1} \rightarrow X_{3}$ family as a distribution to smooth the MLE for the $X_{4} \rightarrow X_{6}$ family's CPT towards. We refer to the $X_{1} \rightarrow X_{3}$ family as a progenitor for the $X_{4} \rightarrow X_{6}$ family, Figure 1b. Specifically, the progenitor for a family with $P$ parents $\left\{X_{i_{1}}, \ldots, X_{i_{p}}\right\}$ and child $X_{j}$ is a family with parents $\left\{X_{\hat{i}_{1}}, \ldots, X_{\hat{i}_{p}}\right\}$ and child $X_{\hat{j}}$ such that, $1 \leq p \leq P$, is the h-parent of $X_{i_{p}}$ and $X_{\hat{j}}$ is the h-parent of $X_{j}$ The $X_{4} \rightarrow X_{6}$ family is referred to as the $X_{1} \rightarrow X_{3}$ family's progeny.

The progenitor and progeny for a family $X_{i}$ are denoted $\vec{\Lambda}^{x_{i}}$ and $\vec{\Lambda}_{x_{i}}$, respectively. As family $X_{i}$ 's progenitor is also a family, it will also have a progenitor. This family is $X_{i}$ 's progenitor's progenitor, or more simply, $X_{i}$ 's second-order progenitor. We denote family $X_{i}^{\prime}$ s $p^{t h}$-order progenitor as $\vec{\Lambda}^{p, x_{i}}$ and the $p^{\text {th }}$ order progeny as $\vec{\Lambda}_{p, X_{i}}$. A family's $0^{\text {th }}$-order progenitor, $\vec{\Lambda}^{0, X_{i}}$ refers to the family itself.

For the moment, we assume the topology of the hierarchy is a forest and all of the nodes within a single BN family are in the same h-level (we return to the more complex case in the next subsection). In this case, determining a family's progenitor is straight-forward. The child in $\vec{\Lambda}^{x_{i}}$ is $\Lambda^{x_{i}}$ and the parents in $\vec{\Lambda}^{x_{i}}$ are $\Lambda^{p_{a}\left(x_{i}\right)}$. Once a family's progenitor and higher-order progenitors have been determined, the MLE for the family can be smoothed towards their MLEs.

The MLE for a BN's parameters is $\hat{\theta}_{X_{i}, j, k}=N_{i, j, k} / N_{i, j}$, where $N_{i, j, k}$ is the number of times in the data that $X_{i}=k$ when $P a\left(X_{i}\right)=j$ and $N_{i, j}$ is the number of times $\operatorname{Pa}\left(X_{i}\right)$ $=j$. The shrinkage estimate for family $X_{i}^{\prime}$ s CPT is calculated as the linear combination of MLEs of the multiple orders of progenitors of $X_{i \text {. This is done on a }}$ multinomial-by-multinomial basis resulting in the following estimate,

$$
\breve{\theta}_{X_{i}, j}=\sum_{p=0}^{V_{i}} \lambda_{X_{i}, j}^{p} \hat{\theta}_{\left(\bar{\Lambda}^{p, X_{i}}\right), j}+\bar{\lambda}_{X_{i}, j} \theta_{X_{i}, j},
$$

where $V_{i}=$ level $_{\Lambda}\left(X_{i}\right)$ (i.e., the number of h-levels above $X_{i}$ that exist in the hierarchy), $\bar{\theta}_{X_{i}, j}$ is the uniform multinomial s.t. $\bar{\theta}_{X_{i}, j, k}=1 / r_{i} . \bar{\lambda}_{X_{i}, j}$ is the uniform estimate's mixture weight, and $\sum_{p=1}^{V_{i}} \lambda_{X_{i}, j}^{p}+\bar{\lambda}_{X_{i}, j}=1$. Inclusion of the uniform estimate prevents the need for any Laplace smoothing when calculating the MLE estimates. If the arity of hierarchically related RVs differs, a mapping function must be provided that maps elements in the domain for one RV into elements in the domain for the other RV (in essence, allowing the CPTs for RVs with different arities to have the same number of elements). 


\subsection{Progenitor Complications}

In a BN family, one node can only be the family's child xor one of the family's parents. Progenitors do not necessarily conform to these constraints. For instance, take the hierarchy in Figure 1a. If the progenitor of family $X_{4} \rightarrow X_{5}$ was needed (Figure 1c) three possible progenitors exist, $X_{1} \rightarrow X_{3}, X_{1} \rightarrow X_{2}$ and $X_{1} \rightarrow X_{1}$. This is because $X_{5}$ has three h-parents, $X_{1}, X_{2}$ and $X_{3}$.

The $X_{1} \rightarrow X_{1}$ family is invalid as it is a cyclic relationship. However, we are not proposing to add progenitors into a BN's topology. Progenitors are only used to generate distributions to be shrunk towards. The CPT for this (invalid) family would be the $P\left(X_{1} \mid X_{1}\right)$ distribution, which is a valid, though uninteresting, identity matrix. We leave this distribution in the shrinkage process, though it could likely be omitted.

A similar event occurs when a family's progenitor contains two parents that are not unique. E.g., consider the family composed of the links $X_{1} \rightarrow X_{2}$ and $X_{1} \rightarrow X_{2}$ (two identical links). This does not represent a legal BN topology as only a single link is allowed to connect two unique nodes. The CPT for this (invalid) family is $P\left(X_{2} \mid X_{1}, X_{1}\right)$, which contains two sets of multinomials: the $P\left(X_{2} \mid X_{1}=\alpha, X_{1}=\alpha\right)$ multinomials, which are valid and equal $P\left(X_{2} \mid X_{1}=\alpha\right)$; and the $P\left(X_{2} \mid X_{1}=\alpha, X_{1}=\beta\right), \alpha \neq \beta$, multinomials, which contain untrainable parameters (as no data point could have $X_{1}=\alpha$ and $X_{1}=\beta$ simultaneously). Recall that we employ shrinkage at the multinomial level. Thus, the valid multinomials may be incorporated into the shrinkage process normally and the invalid multinomials are discarded.

When a node in the hierarchy has more than a single h-parent, i.e., when the topology is a trellis and not a forest, any BN family the node participates in will have multiple progenitors, Figure 1c. Each of those progenitors may in turn have multiple progenitors, resulting in a possibly exponential increase in the number of higher-order progenitors for a family. In our experiments, it was not uncommon to see thousands of progenitors for a single family.

Theoretically, the MLEs for each of these progenitors could be computed and used in a shrinkage process. This is not computationally tractable, particularly for dense trellis hierarchies. Thus, when a family has more than one $p^{\text {th }}$-order progenitor, we create a new meta-progenitor that represents a merging of all the $p^{\text {th }}$-order progenitors. The meta-progenitor's child is a new RV representing the union of all the $p^{\text {th }}$-order progenitors' children. Each of the meta-progenitor's parents are new $\mathrm{RV}$ s representing the union of the $p^{\text {th }}$-order progenitors' corresponding parents.

The value for the new RVs given a data point is determined by a weighted voting procedure. E.g., if the RVs $X_{1}, X_{2}$ and $X_{3}$ are being merged into a new $\mathrm{RV} \dot{X}$, if $X_{1}=$ $1, X_{2}=1$ and $X_{3}=2$ in a given data point, then $\dot{X}=1$ for that data point since that was the most common value seen by the RVs merged into $\dot{X}$. Ties were broken arbitrarily, but occurred rarely due to weighted votes. The MLE for the multinomials in the meta-family's CPT can be computed and incorporated into the shrinkage estimate as a $\hat{\theta}_{\left(\bar{\Lambda}^{p}, X_{i}\right), j}$ term in Equation 2. In many domains, the hierarchy relating the RVs will be a forest (or even a tree) and this merging process will not be required.

Another slight complication occurs when a family contains nodes from different $\mathrm{h}$ levels. E.g., assume the progenitor of family $X_{1} \rightarrow X_{6}$ is needed. This family contains 
a node, $X_{0}$, at the highest level of the hierarchy given in Figure 1a, but also a node, $X_{3}$, at the second highest level. When constructing the progenitor of this family, the hparent of any node at the highest level is set to be the node itself. Thus, the progenitor of family $X_{0} \rightarrow X_{3}$ is $X_{0} \rightarrow X_{0}$. This family does not have any progenitors as all nodes are at the highest level of the hierarchy.

\subsection{Estimating Shrinkage Coefficients}

Given the MLEs, $\hat{\boldsymbol{\theta}}=\left\{\hat{\theta}_{\left(\bar{\Lambda}^{0, X_{i}}\right), j}, \hat{\theta}_{\left(\vec{\Lambda}^{1, X_{i}}\right), j}, \ldots, \hat{\theta}_{\left.\overline{(}^{V_{i}, X_{i}}\right), j}, \bar{\theta}_{X_{i}, j}\right\}$, the mixture weights, $\lambda=\left\{\lambda_{X_{i}, j}^{0}, \lambda_{X_{i}, j}^{1}, \ldots, \lambda_{X_{i}, j}^{V_{i}}, \bar{\lambda}_{X_{i}, j}\right\}$, are set to maximize the empirical likelihood of the learned BN. I.e., the estimates are set to those that result in the highest likelihood possible given a set of left out data, $\tilde{D}$ (the need for left-out data will be addressed shortly). When estimating the multinomial $\theta_{X_{i}, j}$, only the subset of data points in $\tilde{D}$ where $\operatorname{Pa}\left(X_{i}\right)=j$ is needed. We refer to this subset as $\tilde{D}^{\prime}$.

Maximizing the likelihood of the learned BN given $\tilde{D}$ can be done by selecting the parameters that match the MLE of the BN given $\tilde{D}$ as closely as possible. Thus, the shrinkage mixing coefficients are chosen by minimizing the following function: $\lambda=\arg \min _{\lambda^{\prime}}(C P T L o s s(\breve{\theta}, \tilde{\theta}))$, where $\breve{\theta}$ is the shrinkage estimate computed via Equation 2, $\tilde{\theta}$ is the MLE given the left-out data and $\operatorname{CPTLoss}(\breve{\theta}, \tilde{\theta})=$ $\sum_{j=1}^{q_{i}} \sum_{k=1}^{r_{i}}\left|\breve{\theta}_{X_{i}, j, k}-\tilde{\theta}_{X_{i}, j, k}\right|$.

As the data likelihood of a mixture of multinomials is a convex function [14], a simple version of the EM algorithm can be used to find the optimum value for mixture coefficients. The algorithm is given in Figure 2.

This algorithm assumes that each data point, $d \in \tilde{D}^{\prime}$, is drawn from a mixture of multinomials. I.e., a die roll determines which multinomial to use, and another roll determines what value $X_{i}$ takes on given the multinomial. This algorithm selects mixture coefficients such that the linear combination of MLE estimates on the training data is as close to the MLE estimate for the left out data as is possible.

Two issues arise. First, requiring the algorithm to use left-out data inefficiently uses the available data and second, the set of left-out data may be biased (as it represents a relatively small amount of data). To address these issues, a form of cross validation is employed. During each stage of the cross validation, a different section of the training data is set aside as the left-out data. Mixing coefficients are learned that simultaneously maximize the fit of the shrinkage estimates to the left-out estimates across all folds. I.e., $\left.\lambda=\arg \min _{\lambda^{\prime}}\left(\sum_{v=1}^{\# \text { folds }} \operatorname{CPTLOSs}\left(\breve{\theta}_{v}, \tilde{\theta}_{v}\right)\right)\right)$, where $\breve{\theta}_{v}$ and $\tilde{\theta}_{v}$ are the shrinkage estimates and left-out MLE for the $v^{\text {th }}$ fold, respectively. Thus, all the data is used both to draw MLE from and to train the coefficients.

The EM algorithm usually converged in hundreds of iterations. The convergence rate can be dramatically increased (usually to 10 or fewer iterations) by making significantly larger steps at each iteration than proposed by EM. See [4] for details.

\section{Results}

Experiments are performed on two domains. The first is a simulated domain where ground truth is known and controllable. Data with varying degrees of noise was 


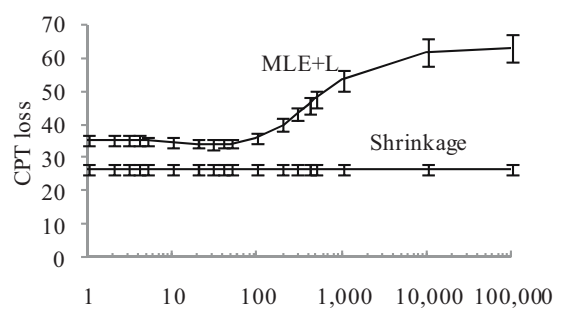

a) Laplace Smoothing Const, $\alpha$

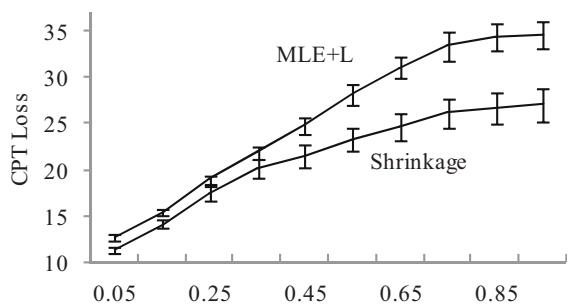

b) Generative Noise parameter, $\rho$

Fig. 3. Simulated data results

generated and the two estimators, MLE+L versus shrinkage, were tested to see how closely they could estimate the underlying generative parameters (comparisons to MLE without any smoothing are not possible as multinomials containing zero-valued probabilities will likely exist and yield invalid data log-likelihoods).

The second domain is a challenging real-world neuroimaging domain consisting of functional magnetic resonance imaging data (fMRI) datasets. Ten datasets were collected on patients that were either healthy, demented or suffered from schizophrenia $[2,6,12]$. For each group of patients, a BN was learned that modeled the correlations among approximately 150 regions in the brain. fMRI data is temporal and time was explicitly represented in the BN structures. Such BNs are referred to as dynamic Bayesian networks. See [4] for further modeling details.

\subsection{Simulated Data}

In all simulated experiments, a single hierarchy, $\Lambda$, over $\mathrm{RVs} X=\left\{X_{1}, \ldots, X_{57}\right\}$, is created with three h-levels containing 3,9 and 45 nodes. A data-generating dynamic Bayesian network (DBN), $G$, is constructed with nodes $\left\{X_{1}^{t}, X_{1}^{t+1}, \ldots, X_{57}^{t}, X_{57}^{t+1}\right\}$. Each $X_{i}^{t+1}$ node is given one, two or four random parents (depending on the experiment) from the $\boldsymbol{X}^{t} \mathrm{RV}$ s. Each of these links correspond to a temporal correlation and the data points constructed are actually time series. The correlational strength for each random link can be quantified with a normalized mutual information score (NMIS) and CPTs consistent with NMIS are randomly generated. The method used to generate such CPTs is outside the scope of this paper, see [4] for more details. We refer to the distribution of generated CPTs as $P\left(\theta_{i} \mid \boldsymbol{M}\right)$ where $\boldsymbol{M}$ is the set containing one NMIS for every parent in the $X_{i}$ family. If $X_{i}$ contains no parents, $\boldsymbol{M}$ is a value indicating the amount of information in $P\left(X_{i}\right)$.

The CPTs for families with no progenitors $\left(X_{1}, X_{2}\right.$ and $\left.X_{3}\right)$ are generated from the $P\left(\theta_{i}^{B} \mid\{c\}\right)$ distribution, yielding families that have increasingly strong correlations as $c$ increases. The CPTs for families with progenitors are initially set as copies of their progenitor's CPTs. Then $h$ random locations in the progeny's CPTs are chosen (with replacement) and a random amount of probability mass between 0 and 0.1 is moved into or out of the random location. The larger $h$ is, the less similar the CPTs between hierarchically related families are, and the less helpful shrinkage should be. 
Each simulated data point is not drawn directly from $G$. Instead, the $\ell^{\text {th }}$ data point is drawn from a new BN, $G^{\ell}$, whose structure is identical to $G$ but whose CPTs are noisy versions of $G^{\prime}$ 's CPTs. The CPTs in $G^{\ell}$ are initially set as copies of the CPTs in $G$. For each CPT, $\theta_{i}^{G^{\ell}}$, a random tuple, $\langle j, k\rangle$, is chosen. The probability mass assigned to $\theta_{i, j, k}^{G^{\ell}}$ is increased by a value chosen between 0 and $\rho, 0<\rho<1$, and the multinomial is renormalized. As $\rho$ increases, the amount of noise introduced into $G^{\ell}$ increases and the more distinct each generated dataset is from $G$. The task is: given a set of $L$ data points, $\left\{d_{1}, d_{2}, \ldots, d_{L}\right\}$, learn parameters that minimize the CPT loss between learned CPTs and the original CPTs in $G$ (results were qualitatively similar across multiple noise models) .

\subsubsection{Learning the Generative BN's Parameters}

Laplace smoothing adds an additional hyper-parameter, $\alpha$, into the learning process that controls how much the MLE+L is smoothed towards a uniform distribution. The first experiment compares MLE+L with varying Laplace smoothing constants to shrinkage estimates. Four simulated data points, each with 1000 time points, are generated from four generating BNs, $\left\{G^{1}, G^{2}, G^{3}, G^{4}\right\}$. Each family in the generative BNs contains a single random parent; $c$ equals $0.05 ; \rho$ equals 0.1 and $h$ equals 2 . At these settings, families in the generating BNs have CPTs that are moderately correlated with their progenitors' CPTs, the parents and children in families are loosely correlated and the noise added to each dataset is significant, but not overwhelming. These settings are arbitrary and the qualitative features of the results are maintained with a wide ranges of values for $c, \rho$ and $h$.

Figure 3a gives the results comparing the estimators' ability to reconstruct $G$ 's CPTs across varying smoothing constants. The tests were repeated 20 times and confidence intervals representing one standard deviation above and below the mean are plotted. Shrinkage does not require a Laplace smoothing constant and is provided as a reference line. Even when the optimal Laplace smoothing constant is derived empirically, shrinkage results in CPTs with lower loss. I.e., shrinkage estimates result in parameters more similar to the generative BN's parameters before noise was added.

Figure $3 \mathrm{~b}$ lists the results for varying degrees of noise. As the amount of probability mass randomly perturbed in $G_{l}$ through $G_{4}$ 's CPTs increases, MLE+Ls become more and more skewed. This is because they simply average the noisy observations into their estimates. Shrinkage estimates are less affected by the increasing noise and are capable of learning estimates with lower CPT loss.

The shrinkage estimates take advantage of the correlation between a family's CPT and the family's progenitor's CPT. The strength of this correlation will vary in real-world domains and may even be negligible. We've performed experiments with decreasing levels of correlation between progenitors' CPTs. As the correlation decreases (i.e., as $h$ increases), the improvement gained by using shrinkage also decreases, but even when the progenitors' CPTs are drawn independently of their progenies' CPTs, shrinkage performs significantly $(p<0.05)$ better than MLE+L. This agrees with Stein's observations on shrinkage estimators [16].

\subsection{Neuroimaging Data}

We also estimated CPTs from the neuroimaging datasets with artificially introduced noise. Noise was added as random changes to the values of RVs in the training data. 


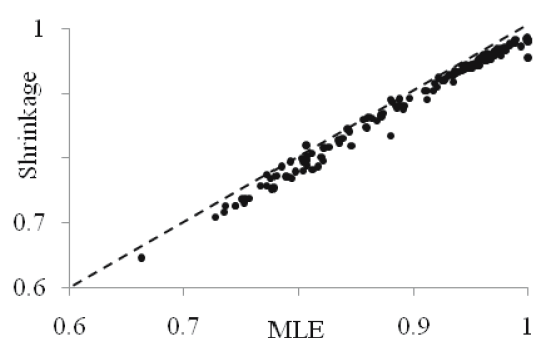

a) Two parents (64 parameters) per family

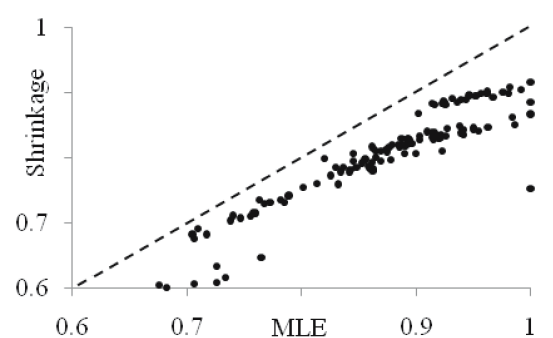

b) Four parents (1024 parameters) per family

Fig. 4. Negative log likelihood of left-out data. The results from the ten fMRI datasets are plotted on each graph. The likelihood values have been normalized to range from zero to 1 . Smaller values indicate higher data-likelihood, thus points under the line indicate left-out likelihood was higher for shrinkage estimates.

As in the simulated domain, shrinkage estimates were capable of estimating CPTs that were less affected by the noise and closer to the CPTs for the noiseless datasets (results were omitted due to space constraints, see [4] for additional details).

As shrinkage estimates result in lower risk than MLE $+\mathrm{L}$ estimates, the data likelihood of BNs given left-out data (of the same class) should also be higher with shrinkage estimates on average than with MLE estimates. We demonstrate that this is the case for the ten fMRI datasets via cross validation experiments.

For each cross-validation fold, the fMRI datasets are split into two portions. Approximately $80 \%$ of a data set is used for training and $20 \%$ is used as left-out data. BNs are then learned on the training data using either MLE+L or shrinkage estimates. The data-likelihood of the trained BNs given the left out data is then calculated.

Figure 4 lists the results for the ten datasets. The graphs plot the negative log likelihood (normalized across datasets) of BNs given the left-out datasets. Points under the line indicate that shrinkage resulted in higher log-likelihoods (lower negative log-likelihood) than MLE+L. As the number of parameters grow, the benefit of using shrinkage increases. When the average number of parents per node is two, shrinkage estimates result in higher left-out data likelihoods $92 \%$ of the time. That number jumps to $100 \%$ when the average number of parents per node is four. Further, the margin by which shrinkage beats MLE+L on individual left-out datasets also increases with the number of parameters.

Even though left-out generalization clearly improves with shrinkage estimates, it is not necessarily the case that classification accuracy will change. While classification is not a primary goal of this work, we have performed an array of classification experiments using the generative BDe score [10] (which does not require parameterization during the structure search) and the class-discriminative ACL-ML score [3] (which does require parameterization during the structure search).

Differences in classification accuracies due to shrinkage were not significantly changed from MLE+L. Of 590 classification experiments performed (with varying complexities of learned BNs), BNs trained with MLE+L correctly classified 382 datasets $(64.7 \%)$ and BNs trained with shrinkage estimates correctly classified 372 datasets $(63.1 \%)$; a statistically insignificant difference. 


\section{Conclusions}

One component of learning Bayesian networks is the estimation of parameters. A widely employed estimator is the MLE with Laplace smoothing (MLE+L). Stein demonstrated that the MLE was inadmissible in certain cases [16]. Several machine learning modeling techniques were shown to benefit from shrinkage estimates $[1,14]$ by constructing a hierarchy of classes and shrinking a RV's MLE towards the MLE of $\mathrm{RVs}$ in the other classes.

We have proposed a similar means of shrinking a RV's MLE towards the MLEs of other hierarchically related RVs in the same class. Shrinkage estimates are calculated as weighted linear combinations of MLEs where the mixture coefficients are learned via an EM algorithm. To our knowledge, we are also the first to propose using a shrinkage estimator during BN structure search.

We performed experiments on a simulated domain where ground truth was known and controllable as well as on a challenging real-world neuroimaging domain. On the simulated data, we demonstrated that even when the ideal Laplace smoothing constant is known, shrinkage estimates allow for better parameter estimates in the presence of noisy data. As the amount of noise increased, shrinkage's benefit increased. This was true (though diminished) even if the RVs in the hierarchy were independent, which agrees with Stein's [16] original work with shrinkage.

On the fMRI data, we also found that shrinkage was capable of estimating fMRI parameters more effectively than MLE $+\mathrm{L}$ given noisy versions of the fMRI data. As the level of noise increased, the performance of shrinkage estimates also increased. We further showed that using shrinkage estimates increases the generalization of the learned BNs by increasing the likelihood of data points (of the same class) left out of training process. This increase was found not to be caused solely by shrinking towards a uniform distribution (a type of empirical Laplace smoothing), but that shrinkage towards other distributions found in the data was advantageous. This is in agreement with [1, 14]. Finally, we found that the application of shrinkage estimates did not diminish the classification performance of learned BNs.

Future work involves applying these shrinkage techniques to domains that do not have a preexisting hierarchy among their RVs. For such domains, a synthetic hierarchy could be constructed by aggregating sets of RVs together into new RVs. This raises yet unanswered questions such as which RVs should be aggregated together, how many levels the synthetic hierarchy should have, what aggregation should be used, etc. Given the results on the fMRI data-a domain in which hierarchically related brain regions often have significantly different functional behaviors - we believe our shrinkage estimates will perform well in general. Indeed, in preliminary experiments where the hierarchy used to guide shrinkage was randomized, shrinkage estimates still yielded better estimates than MLE+L.

\section{Acknowledgments}

We would like to thank the anonymous reviewers for their valuable feedback and Dr. Randy Buckner, Dr. Kent Kiehl, Dr. Vincent P. Clark and The MIND Institute for providing access to their neuroimaging datasets. This work was funded by grants DA012852, NIDA, NIH; 1R01MH076282-01, NIMH; DE-FG02-99ER62764, DOE. 


\section{References}

1. Anderson, C., Domingos, P., Weld, D.: Relational Markov models and their application to adaptive web navigation. In: International Conference on Knowledge Discovery and Data Mining, pp. 143-152 (2002)

2. Buckner, R.L., Snyder, A., Sanders, A., Marcus, R., Morris, J.: Functional Brain Imaging of Young, Nondemented, and Demented Older Adults. Journal of Cognitive Neuroscience 12, 24-34 (2000)

3. Burge, J., Lane, T.: Class-Discriminative Dynamic Bayesian Networks. In: ICML, Bonn, Germany (2005)

4. Burge, J.: Learning Bayesian Networks from Hierarchically Related Data with a Neuroimaging Application. Ph.D. Dissertation. Computer Science. University of New Mexico, New Mexico (2007)

5. Carlin, B.P., Louis, T.A.: Bayes and Empirical Bayes Methods for Data Analysis. Chapman \& Hall, London (1996)

6. Clark, V.P., Friedman, L., Manoach, D., Ho, B.C., Lim, K., Andreasen, N.: A collaborative fMRI study of the novelty oddball task in schizophrenia: Effects of illness duration. Society for Neuroscience Abstracts 474.474 (2005)

7. Friedman, N., Geiger, D., Goldszmidt, M.: Bayesian Network Classifiers. Mach. Learn. 29, 131-163 (1997)

8. Grossman, D., Domingos, P.: Learning Bayesian Network Classifiers by Maximizing Conditional Likelihood. In: International Conference on Machine Learning, pp. 361-368 (2004)

9. Gupta, A.K., Ehsanese Saleh, A.K.M.: Estimation of Multinomial Probabilities under a Model Constraint. Journal of Multinomial Estimation 58, 151-161 (1996)

10. Heckerman, D., Geiger, D., Chickering, D.M.: Learning Bayesian networks: The combination of knowledge and statistical data. Machine Learning 20, 197-243 (1995)

11. James, W., Stein, C.: Estimation with quadratic loss. In: Berkeley Symposium on Mathematical Statistics and Probability, vol. 1, pp. 361-379. University of California Press (1960)

12. Kiehl, K.: An event-related functional magnetic resonance imaging study of an auditory oddball task in schizophrenia. Schizophrenia Research 48, 159-171 (2001)

13. Lam, W., Bacchus, F.: Learning Bayesian Belief Networks. An Approach Based on the MDL Principle. Computational Intelligence 10, 269-293 (1992)

14. McCallum, A., Rosenfeld, R., Mitchell, T., Ng, A.Y.: Improving Text Classification by Shrinkage in a Hierarchy of Classes. In: International Conference on Machine Learning, pp. 359-367 (1998)

15. Pearl, J.: Fusion, Propagation, and Structuring in Belief Networks. AI 29, 241-288 (1986)

16. Stein, C.: Inadmissibility of the usual estimator for the mean of a multivariate normal distribution. In: Third Berkeley Symposium on Mathematical Statistics and Probability, vol. 1, pp. 197-206. University of California Press (1955) 